\title{
MEDICIÓN DE LA CULPA EN LA RELACIÓN DE PAREJA
}

\author{
THE GUILT MEASURING ON RELATIONSHIP \\ Elizabeth Alvarez Ramírez \\ Universidad Nacional Autónoma de México \\ Mirna García Méndez \\ Universidad Nacional Autónoma de México \\ Sofía Rivera Aragón \\ Universidad Nacional Autónoma de México
}

\begin{abstract}
Resumen: El objetivo del estudio fue validar una escala para medir la culpa en la relación de pareja. El estudio se llevó a cabo en dos etapas: primero, se realizó un análisis exploratorio mediante redes semánticas, con 200 participantes, se obtuvieron indicadores y categorías asociados a la culpa. En base a estos resultados se realizó una escala tipo Likert y en la segunda fase, se obtuvo la validez de constructo con una muestra de 850 participantes de la Ciudad de México. La escala de culpa en la relación de pareja quedó integrada por un total de 72 reactivos, distribuidos en cinco subescalas (significado, causas, consecuencias, revaloración y efectos de la culpa), con un alpha de Cronbach global de .929 y una varianza explicada de 63.13. La estructura factorial se comprobó a partir de ecuaciones estructurales, se obtuvieron índices de bondad de ajuste adecuados, por lo que se puede concluir que es un instrumento válido.
\end{abstract}

Palabras Clave: conflicto, culpa, relación de pareja, emociones

\begin{abstract}
This study was aimed at validating a scale for assessing guilt in a relationship. The study was conducted in two stages: first, an exploratory analysis — based on 200 subjects - using semantic networks was performed. Guilt-related conceptual categories and indicators were obtained. Based on these findings, a Likert-scale was constructed. On a second stage, the construct validity of the scale was assessed using a sample of 850 participants from Mexico City. The final scale comprises a total of 72 items, distributed in five subscales (meaning, causes, consequences, and revaluation, and effects of guilt), with a Cronbach's alpha of .929 overall and accounts for $63.13 \%$ of the variance. The factorial structure was tested via structural equations; appropriate good of fitness indices were obtained. It can be concluded that this scale is a valid instrument.
\end{abstract}

Keywords: conflict, guilt, couple, emotion

\section{Introducción}

En la década de los 90 la culpa fue considerada una emoción social y negativa en su origen, que surgía a partir de que el individuo se siente responsable del sufrimiento del otro (Frijda, 1994). Sin embargo, hay autores que mencionan que la culpa tiene un efecto positivo en las relaciones, debido a que favorece las conductas reparadoras, a través de las cuales se busca enmendar el daño y aumentar el bienestar del otro (Tangney \& Dearing, 2004; Tangney \& Fischer, 1995) con esta premisa se asume que la culpa tiene una función social, debido a que facilita la interacción de los individuos a partir de la realización de conductas apropiadas, que a su vez influyen en las relaciones interpersonales haciéndolas más eficientes.
Etxebarria y Apodaca (2008) refieren que desde los años 60 la culpa fue descrita como una ansiedad experimentada ante el temor de la pérdida del amor de personas cercanas o por sentirse responsables del daño causado a un ser amado. Surge en la persona que siente culpa, la necesidad de reparar el daño debido a la sensación de que actuó de manera inapropiada con el otro. En el análisis que hacen estos autores sobre los componentes de la culpa, encuentran por un lado el ansioso agresivo, relacionado con el temor de la perdida y por otro, el componente empático que funciona como un generador de conductas prosociales, que fomentan la reparación y actitudes positivas dirigidas a enmendar el daño, apegándose a las normas sociales preestablecidas (Martorelli, González, Ordóñez \& Gómez 2011). Al respecto sugieren que estas conductas guían 
a los individuos para apegarse a las normas sociales preestablecidas. Cabe resaltar que la culpa surge principalmente en experiencias de tipo interpersonal cercanas (Etxebarria \& Apodaca, 2008).

En este sentido, la culpa puede verse como un proceso, en el que intervienen elementos cómo: valoraciones cognitivas, afectivas y comportamientos que discrepan de la escala de valores establecida por la cultura (Etxebarria, 1999; Zabalegui, 1997). Al respecto, Álvarez (1996) sugiere que la culpa inicia con la trasgresión de una norma o con una mala acción hacia otra persona, además explica que si la persona se atribuye la responsabilidad del sufrimiento del otro, experimentará una sensación desagradable que lo motivará a la reparación o al autocastigo, con la finalidad de recuperar la confianza social y aliviar su malestar, y del mismo modo mantener su estatus quo.

Respecto a las situaciones que generan culpa en las relaciones, Baumeister, Reis y Delespaul (1995) reportan que las causas más frecuentes son el descuido de las relaciones interpersonales, en especial la de pareja, y el fallo en el cumplimiento de obligaciones o compromisos con alguien;. como fuentes secundarias, mencionan en orden de mayor a menor frecuencia, la infidelidad sexual o emocional, ser agresivo, el descuido de los estudios o el trabajo, traicionar la confianza de un amigo, comer demasiado, la implicación en alguna desgracia ajena, mentir, derrochar dinero y descuidar el ejercicio.

Posteriormente, Etxebarria y Pérez (2003) reafirman los anteriores hallazgos, y concluyen que la culpa se origina principalmente en situaciones de carácter interpersonal y en menor grado, en torno a problemas de autorregulación, es decir, la culpa modera en el individuo el sentido del bien y el mal, al diferenciar entre la buena y la mala conducta, lo que favorece la autorregulación del comportamiento y a su vez permite crear y mantener un equilibrio personal y social en las relaciones (Rojas Marcos, 2009).

En diversas áreas de investigación cómo la psicología evolutiva y social, se ha hecho hincapié en las funciones adaptativas de la culpa en relación con la conducta moral, la adaptación social (Barret, 1995; Baumeister, Stillwell \& Heartherton, 1994; Hoffman, 1998; Tangney, 1990, 1995a, 1995b; Tangney, Burggraf \& Wagner, 1995) y su importancia en el proceso de socialización, pues se considera un motivador primario que orienta a las personas a desarrollarse y relacionarse de acuerdo con las normas establecidas en cada cultura (Reidl \& Jurado, 2007).

El arrepentimiento y la preocupación empática hacia a otros, son resultado de actuar de manera incorrecta e incluso el pensar que no se hizo lo necesario por evitar el sufrimiento de alguien más (Benetti-Mcquoid \& Bursik, 2005). Este efecto se hace evidente al generar conductas dirigidas a compensar al otro, tales como el pedir perdón, la autocorrección, el retribuir y reparar el daño (Etxebarria, Pascual \& Conejero, 2010; Hoffman, 2002).

Particularmente en el contexto de la relación de pareja, Ojeda, Torres y Moreira (2010) mencionan que las normas sociales o estilos de interacción ayudan a que los integrantes de la díada se conozcan e infieran lo que es correcto e incorrecto para su buen funcionamiento. Entonces, el quebrantar estas normas establecidas, puede generar una experiencia de culpa en el trasgresor, por faltar al compromiso hacia su pareja.

La culpa representa un papel importante en la socialización y las interacciones cercanas de todo individuo, en tal sentido, resulta oportuno y trascendental su estudio y medición. Autores interesados en esta temática han diseñado diversos instrumentos sobre la evaluación de la culpa (ver Tabla 1), la mayoría se enfoca principalmente en dos áreas: la que evalúa el estado emocional que se experimenta en el momento (estado); y la que mide rasgos emocionales o disposiciones a la culpa (rasgo).

A pesar de que los instrumentos mencionados cuentan con varianza y confiabilidad aceptables, fueron diseñados en otras culturas. En México, Fernández de Ortega y Reidl (2005) estandarizaron la escala de Kugler y Jones (1992) que evalúa la culpa como rasgo, estado y los estándares sociales, empero, a pesar de considerar las interacciones interpersonales, no está dirigida a las relaciones amorosas.

Con base en la literatura se observó que la culpa tiene un efecto en las relaciones de pareja, se considera importante profundizar en el conocimiento de la influencia de la culpa en la interacción de pareja. En consecuencia, el objetivo de este estudio fue elaborar una escala multidimensional para evaluar las dimensiones que integran la culpa en la relación de pareja. Para lograr este propósito, el estudio se llevó a cabo en dos etapas: la primera consistió en un análisis exploratorio realizado a través de la técnica de redes semánticas, con base en que, a través del lenguaje cotidiano de los individuos 
Tabla 1. Escalas de culpa y sus dimensiones

\begin{tabular}{|c|c|c|}
\hline Escalas & Evalúa & Dimensiones \\
\hline $\begin{array}{l}\text { Subescala de culpa del inventario de } \\
\text { hostilidad (Buss-Durkee, 1957) }\end{array}$ & $\begin{array}{l}\text { Conductas disruptivas que deben ser } \\
\text { castigadas }\end{array}$ & $\begin{array}{l}\text { Sentimientos de ser malo, actuar mal, } \\
\text { remordimientos de conciencia }\end{array}$ \\
\hline $\begin{array}{l}\text { Inventario de culpa (MITS) (Mosher, } \\
\text { 1966) }\end{array}$ & Estado y rasgo de la culpa & $\begin{array}{l}\text { Culpa sexual, culpa hostil, culpa concien- } \\
\text { cia-moralidad, verdadero -falso }(\alpha=.91) \text {. } \\
\text { Resp. Forzada ( } \alpha=.97)\end{array}$ \\
\hline $\begin{array}{l}\text { Índice de culpa percibida (Otterbacher } \\
\& \text { Munz, 1973) }\end{array}$ & Experiencia de culpa inducida y reacción. & Culpa rasgo, culpa estado \\
\hline $\begin{array}{l}\text { Inventario de reacción a la culpa } \\
\text { (Evans, Jessup, \& Hearn, 1975) }\end{array}$ & $\begin{array}{l}\text { Conductas disruptivas en las áreas perso- } \\
\text { nal, social e interpersonal }\end{array}$ & $\begin{array}{l}\text { Conducta disruptiva en las relaciones in- } \\
\text { terpersonales, conducta autodestructiva, } \\
\text { conducta contraria a la moral, conducta } \\
\text { disruptiva no intencional }(\alpha=.52)\end{array}$ \\
\hline $\begin{array}{l}\text { Escala de culpa situacional (SGS) } \\
\text { (Klass, 1987) }\end{array}$ & Anticipación de la culpa inducida & $\begin{array}{l}\text { Culpa daño interpersonal, culpa por } \\
\text { violación de normas, culpa por falta de } \\
\text { autocontrol. }(\alpha=.92)\end{array}$ \\
\hline $\begin{array}{l}\text { Escala de miedo al castigo/necesi- } \\
\text { dad de reparación (Caprara, Manzi \& } \\
\text { Perugini, 1992) }\end{array}$ & $\begin{array}{l}\text { Culpa relacionada con emocionalidad y } \\
\text { agresión. }\end{array}$ & $\begin{array}{l}\text { Miedo al castigo, necesidad de reparación } \\
(\alpha=.91)\end{array}$ \\
\hline $\begin{array}{l}\text { Cuestionario de culpa interpersonal } \\
\text { (IGQ-45 \& IGQ-67) (O'Connor, Berry, } \\
\text { Weiss, Bush, \& Sampson, 1997) }\end{array}$ & Culpa interpersonal & $\begin{array}{l}\text { Sobrevivir a la culpa, culpa por separación/ } \\
\text { disolución, culpa por responsabilidad om- } \\
\text { nipotente, culpa auto-odio IGQ-45 }(\alpha=.79) \\
\text { IGQ-67 }(\alpha=.85)\end{array}$ \\
\hline $\begin{array}{l}\text { Inventario de culpa (GI) (Kugler \& } \\
\text { Jones, 1992) }\end{array}$ & $\begin{array}{l}\text { Experiencias recientes y tendencia a expe- } \\
\text { rimentar culpa }\end{array}$ & $\begin{array}{l}\text { Culpa rasgo, culpa estado, estándares } \\
\text { morales }(\alpha=.89)\end{array}$ \\
\hline Escala de culpa (Berrios et al., 1992) & Aspectos de la culpa y vergüenza & $\begin{array}{l}\text { Culpa; conducta, actitudes; estado de } \\
\text { ánimo, sentimiento de culpa }\end{array}$ \\
\hline $\begin{array}{l}\text { Inventario de culpa relacionado con el } \\
\text { trauma (TRGI) (Kubany et al., 1996) }\end{array}$ & $\begin{array}{l}\text { Culpa relacionada con el trauma en vetera- } \\
\text { nos de Vietnam }\end{array}$ & $\begin{array}{l}\text { Culpa general, estrés, pensamientos de } \\
\text { culpa }(\alpha=.90)\end{array}$ \\
\hline $\begin{array}{l}\text { Imagen corporal culpa y vergüenza } \\
\text { (Thompson, Dinnel, \& Dill, 2003) }\end{array}$ & $\begin{array}{l}\text { Culpa y vergüenza relacionado con imagen } \\
\text { corporal }\end{array}$ & Culpa, vergüenza $(\alpha=.91)$ \\
\hline $\begin{array}{l}\text { Inventario de culpa rasgo-estado y } \\
\text { estándares morales (adaptado por } \\
\text { Fernández de Ortega \& Reidl, 2005) }\end{array}$ & $\begin{array}{l}\text { Experiencias recientes y relacionadas con } \\
\text { la tendencia a experimentar culpa }\end{array}$ & Autoatribución, apego a las reglas $(\alpha=.90)$ \\
\hline
\end{tabular}

se puede conocer la representación de algún fenómeno. Se realizó la recolección de datos respecto a cómo los participantes perciben la manifestación de la culpa en su relación de pareja. Posteriormente, con la información obtenida se elaboraron reactivos que integraron las cinco subescalas de una escala tipo Likert, que fue validada en la segunda etapa, mediante un análisis factorial exploratorio (de componentes principales con rotación ortogonal). Para finalizar se obtuvo la bondad de ajuste de cada una mediante un análisis factorial confirmatorio.

\section{Método}

Etapa I: Elaboración de la escala de culpa, estudio exploratorio. Esta etapa tuvo como objetivo identificar los componentes involucrados en las manifestaciones de culpa, sus causas y consecuencias en la interacción de pareja.

\section{Participantes}

Participaron 100 hombres y 100 mujeres de la Ciudad de México, con un rango de edad de 17 a 60 años $(M=26, D E=7.7)$. Respecto al estado civil, el $79 \%$ eran solteros en pareja, el $17 \%$ casados y el $4 \%$ divorciados; el criterio de inclusión fue que, al momento de la investigación tuviesen como mínimo un año en pareja.

\section{Instrumento}

Se utilizó la técnica de redes semánticas naturales modificadas (Reyes Lagunes, 1993), que consiste en evaluar a través del lenguaje cotidiano el significado semántico y la representación de los conceptos relacionados a la culpa en la relación de pareja. Las seis frases- estímulo se integraron en un cuestionario, dirigidas a evaluar el significado, las 
causas, consecuencias y aspectos cognitivos, conductuales y emocionales de la culpa, estas fueron: "el sentimiento de culpa en mi relación de pareja es...", "La culpa en mi relación de pareja ocasiona...", "Siento culpa con mi pareja cuando...", "Mi pareja siente culpa cuando...", "Cuando siento culpa con mi pareja pienso...", "Cuando siento culpa con mi pareja hago...".

\section{Procedimiento}

El instrumento se aplicó de manera individual en lugares públicos de la zona Oriente de la Ciudad de México, escuelas y centros de trabajo. A las personas que participaron, se les explicó la técnica de redes semánticas, que consiste en escribir de 5 a 10 palabras que describan cada uno de los estímulos presentados. Al finalizar la lista, se les pidió que las jerarquizaran del 1 al 10 de acuerdo a la importancia y la relación con la frase estímulo. Se hizo énfasis en que la información sería confidencial y anónima.

Con la información obtenida, se realizó un análisis de datos a través de frecuencias en el programa Excel, se obtuvo el tamaño de la red (TR), que se refiere al total de las diferentes palabras que emitieron los participantes. El peso semántico (PS), que indica la suma de la ponderación de la frecuencia por la jerarquización asignada por cada individuo. La distancia semántica (DSC), que se refiere al porcentaje que representa cada palabra definidora dentro de la red y el núcleo de la red (NR), que consiste en las palabras definidoras más representativas correspondientes a cada frase estímulo.

A partir de cada núcleo de la red obtenida y a la luz de la teoría revisada, se realizó un análisis cualitativo, a través del cual se identificaron indicadores y a su vez dimensiones del constructo culpa en la pareja. En este procedimiento se pudo observar que algunos indicadores se repetían en varias frases estímulo, lo que indica que evalúan esa característica en más de una dimensión (cognitiva, emocional y conductual).

A continuación se diseñaron categorías en donde se agruparon estos indicadores, por lo que también surgieron categorías repetidas. Por tal motivo, se llegó a la conclusión que sería adecuado elaborar más de una escala con la intención de tomar en consideración la importancia de los indicadores y dimensiones obtenidos. Finalmente, a partir de éstos se elaboraron los reactivos componentes de la escala tipo Likert, dirigida a evaluar las dimensiones de la culpa en la relación de pareja (ver Tabla 2).

Tabla 2. Dimensiones e indicadores de la culpa en la relación de pareja, resultado del estudio exploratorio.

\begin{tabular}{|c|c|c|}
\hline Frases estímulos & Dimensiones & Ejemplos de indicadores \\
\hline $\begin{array}{l}\text { El sentimiento de culpa en mi relación de } \\
\text { pareja es... }\end{array}$ & $\begin{array}{l}\text { Reparación del daño } \\
\text { Nocivo } \\
\text { Frecuente } \\
\text { Rasgo de personalidad } \\
\text { Emoción negativa }\end{array}$ & $\begin{array}{l}\text { Depresivo, destructivo, malo, doloroso, moles- } \\
\text { to, frustrante, desagradable, feo, estresante, } \\
\text { complejo, incomodo, angustia, innecesario, } \\
\text { dañino, enojo }\end{array}$ \\
\hline $\begin{array}{l}\text { La culpa en mi relación de } \\
\text { pareja ocasiona... }\end{array}$ & $\begin{array}{l}\text { Ansiedad } \\
\text { Inseguridad } \\
\text { Conflicto }\end{array}$ & $\begin{array}{l}\text { Tristeza, frustración, conflicto, alejamiento, } \\
\text { ansiedad, angustia, malestar, confusión, des- } \\
\text { confianza, miedo }\end{array}$ \\
\hline $\begin{array}{l}\text { Siento culpa con mi pareja cuando... } \\
\text { Mi pareja siente culpa cuando... }\end{array}$ & $\begin{array}{l}\text { Evitación } \\
\text { Egoísmo } \\
\text { Violencia } \\
\text { Deshonestidad } \\
\text { Conflicto } \\
\text { Hábitos negativos } \\
\text { Poder } \\
\text { Celos }\end{array}$ & $\begin{array}{l}\text { Agredo, no dedico tiempo, miento, peleamos, } \\
\text { infiel/engaño, abandono, no me comprometo, } \\
\text { ignoro, hago enojar, no escucho }\end{array}$ \\
\hline Cuando siento culpa con mi pareja pienso... & $\begin{array}{l}\text { Reparación del daño } \\
\text { Evitación } \\
\text { Malestar } \\
\text { Deshonestidad } \\
\text { Acomodación } \\
\text { Ansiedad }\end{array}$ & $\begin{array}{l}\text { Ignorarlo, remediarlo, platicar, pedir perdón/ } \\
\text { disculpas, compensar, tristeza, enojo, angus- } \\
\text { tia, bienestar, hice mal, reflexionar }\end{array}$ \\
\hline Cuando siento culpa con mi pareja hago... & $\begin{array}{l}\text { Reparación del daño } \\
\text { Evitación, Conflicto } \\
\text { Violencia, Malestar } \\
\text { Afrontamiento } \\
\text { Comunicación }\end{array}$ & $\begin{array}{l}\text { Hablar, compensar, evitarlo, llorar, pedir per- } \\
\text { dón, enfrento, reflexionar, enojo, acercarme, } \\
\text { retroalimentación, tranquilizarme }\end{array}$ \\
\hline
\end{tabular}




\section{Etapa II. Análisis psicométrico}

\section{Participantes}

La muestra fue no probabilística de 850 participantes voluntarios, 373 hombres y 477 mujeres residentes de la Ciudad de Méxi$\mathrm{co}$, con un rango de edad de 17 a 65 años $(M=31, D E=11.52)$, el estado civil que reportaron fue : $40 \%$ casados, $18 \%$ en unión libre y $42 \%$ solteros en pareja. La escolaridad fue: primaria $1 \%$, secundaria $15 \%$, técnico $12 \%$, preparatoria $28 \%$, licenciatura $40 \%$ y posgrado $4 \%$. En cuanto al número de hijos la mayoría reportó $2(M o=2)$. El criterio de inclusión fue que los participantes al momento de la investigación tuviesen mínimo un año en su relación de pareja.

\section{Procedimiento}

Los participantes se localizaron en centros de trabajo y en hogares. Se contactaron de manera individual y se les explicó que se trataba de una investigación con fines científicos. A quienes accedieron a participar voluntariamente, se entregó el instrumento y se pidió que firmaran el consentimiento informado; se enfatizó en el anonimato y confidencialidad.

\section{Instrumento}

Con los resultados de las redes semánticas, se elaboró una escala Likert con seis intervalos de respuesta; 1 = totalmente en desacuerdo a 6 = totalmente de acuerdo. La escala se integró por 164 reactivos distribuidos en cinco subescalas: Significado de culpa (31 reactivos), Causas de la culpa (35 reactivos), Consecuencias de la culpa (43 reactivos), Revaloración (18 reactivos) y Efectos de la culpa (37 reactivos).

\section{Resultados}

Se procedió al análisis psicométrico para los reactivos de cada una de las cinco subescalas. Se llevaron a cabo las siguientes pruebas: análisis de frecuencia para conocer la distribución de las opciones de las respuestas, normal o sesgada. La discriminación de los reactivos con la prueba $t$ de Student para grupos extremos con la finalidad de detectar diferencias entre los grupos. La correlación ítem total para identificar el grado de asociación entre los reactivos. Análisis de confiabilidad (Alpha de Cronbach) general y por factor.
Posteriormente, se efectuó un análisis factorial con rotación ortogonal para cada subescala, con la intención de determinar en cuantos factores se agrupan los reactivos. Se eligieron los reactivos con cargas factoriales mayores o iguales a .40 , con valores propios mayores a 1. Una vez obtenidos los factores, se obtuvo el Alpha de Cronbach. Una última prueba realizada fue la correlación producto-momento de Pearson, para establecer la correlación entre los factores obtenidos en cada factorial.

Subescala significado de culpa: la Tabla 3 muestra la solución factorial para la subescala significado de culpa. Se observa que quedó conformada por 20 reactivos distribuidos en 4 factores, que explican el $51.454 \%$ de la varianza total y una Alpha de Cronbach global de 0.842 , por factor oscilan de 0.639 a 0.832 .

Tabla 3. Reactivos y factores de la subescala significado de culpa

\begin{tabular}{lcccc}
\hline \multicolumn{4}{c}{ "Sentir culpa en mi relación de pareja es..." } \\
\hline Reactivos & \multicolumn{4}{c}{ Factores } \\
\hline 5. Molesto & .762 & & 3 & 4 \\
10. Desagradable & .737 & & & \\
8. Dañino & .718 & & & \\
3. Malo & .661 & & & \\
14. Frustrante & .651 & & & \\
13. Complejo & .549 & & & \\
1. Deprimente & .531 & & & \\
18. Deshonesto & & .753 & & \\
21. Injusto & & .700 & & \\
24. Tonto & & .597 & & \\
17. Contradictorio & & .586 & & \\
20. Desmoralizante & & .550 & & \\
30. Castigo & & & .785 & \\
31. Remordimiento & & & .712 & \\
26. Ansiedad & & & .647 & \\
15. Reparar el daño & & & & .680 \\
11. Empático (ponerse en & & & & .654 \\
lugar del otro & & & & \\
9. Humano & & & & \\
19. Disculparse & & & & \\
7. Conciliador & & & & \\
\hline Número de reactivos & 7.25 & 1.19 & 1.40 & 1.10 \\
Varianza explicada & 27.67 & 10.36 & 7.11 & 6.32 \\
Alpha de Cronbach & .83 & .73 & .74 & .64 \\
Media & & & & \\
Desviación Estandar & & & & \\
\hline
\end{tabular}


Los factores del significado de culpa se definieron de la siguiente manera: Factor 1. Negativo (7 reactivos), refiere a las atribuciones negativas, resultado de conductas consideradas inadecuadas hacia la pareja. Factor 2. Valores (5 reactivos), es la actitud hacia comportamientos que trasgreden las normas establecidas. Factor 3. Autocrítica (3 reactivos), refiere a la capacidad de distinguir los propios errores y generar pensamientos redundantes que causan malestar psicológico. Factor 4. Preocupación empática (5 reactivos). Se refiere a las acciones encaminadas a corregir los resultados negativos asociados con el daño ocasionado a la pareja.

Subescala causas de la culpa: la Tabla 4 muestra la solución factorial para esta subescala, que se conformó por 16 reactivos agrupados en dos factores que explican el $63.131 \%$ de la varianza total con un Alpha de Cronbach global de 0.947 y Alpha por factor que oscilan de 0.639 a 0.832. De acuerdo a los reactivos agrupados los factores se definieron de la siguiente manera: Factor 1. Falta de compromiso (13 reactivos). Se refiere específicamente a conductas o actitudes dirigidas a mantenerse alejado del compañero y mantener, a pesar de causarle daño.

Tabla 4. Reactivos y factores de la subescala causas de la culpa

"Existe culpa en mi relación de pareja cuando..."

\begin{tabular}{|c|c|c|}
\hline \multirow{2}{*}{ Reactivos } & \multicolumn{2}{|c|}{ Factores } \\
\hline & 1 & 2 \\
\hline 17. Evito escuchar a mi pareja & .782 & \\
\hline 20. Pienso en ser infiel a mi pareja & .780 & \\
\hline $\begin{array}{l}\text { 33. Beso a alguien más que no sea mi } \\
\text { pareja }\end{array}$ & .770 & \\
\hline 18. Daño a mi pareja & .766 & \\
\hline 30. Abandono a mi pareja & .753 & \\
\hline 16. Pienso en otro (a) & .751 & \\
\hline 25. Soy deshonesto con mi pareja & .745 & \\
\hline 21. Manipulo a mi pareja & .736 & \\
\hline 31. Me burlo de mi pareja & .698 & \\
\hline $\begin{array}{l}\text { 10. Hay falta de compromiso en mi } \\
\text { relación de pareja }\end{array}$ & .674 & \\
\hline 27. Soy aprehensivo con mi pareja & .655 & \\
\hline 14. Ayudo poco a mi pareja & .636 & \\
\hline 15. Le dedico poco tiempo a mi pareja & .635 & \\
\hline 1. Hago llorar a mi pareja & & .863 \\
\hline 2. Le grito a mi pareja & & .808 \\
\hline 3. Desilusiono a mi pareja & & .745 \\
\hline Número de reactivos & 13 & 3 \\
\hline Varianza explicada & 55.571 & 7.651 \\
\hline Alpha de Cronbach & .945 & .821 \\
\hline Media & 3.311 & 3.787 \\
\hline Desviación Estándar & 1.46 & 1.54 \\
\hline
\end{tabular}

Factor 2. Violencia (3 reactivos). Todas las acciones que generan culpa por dañar tanto física, emocional y psicológicamente a la pareja.

Subescala consecuencias de la culpa: un tercer análisis agrupó esta subescala en tres factores, que agrupan 14 reactivo, los que explican el $58.913 \%$ de la varianza total con un Alpha de Cronbach global de 0.861 y Alpha por factor entre 0.742 y 0.831 (ver Tabla 5).

Tabla 5. Reactivos y factores de la subescala consecuencias de la culpa

\begin{tabular}{|c|c|c|c|}
\hline \multicolumn{4}{|c|}{ "La culpa en mi relación ocasiona..." } \\
\hline \multirow[t]{2}{*}{ Reactivos } & \multicolumn{3}{|c|}{ Factores } \\
\hline & 1 & 2 & 3 \\
\hline $\begin{array}{l}\text { 11. Discusiones con mi } \\
\text { pareja }\end{array}$ & .774 & & \\
\hline $\begin{array}{l}\text { 9. Decepcionarme de mi } \\
\text { pareja }\end{array}$ & .713 & & \\
\hline $\begin{array}{l}\text { 7. Conflictos con mi } \\
\text { pareja }\end{array}$ & .693 & & \\
\hline $\begin{array}{l}\text { 13. Distanciamiento con } \\
\text { mi pareja }\end{array}$ & .627 & & \\
\hline $\begin{array}{l}\text { 20. Infelicidad con mi } \\
\text { pareja }\end{array}$ & .614 & & \\
\hline $\begin{array}{l}\text { 26. Mala comunicación } \\
\text { con mi pareja }\end{array}$ & .593 & & \\
\hline $\begin{array}{l}\text { 38. Que sienta desespe- } \\
\text { ración }\end{array}$ & & .760 & \\
\hline 43. Tensión & & .722 & \\
\hline 36. Frustración & & .722 & \\
\hline 39. Sentirme cansado & & .695 & \\
\hline $\begin{array}{l}\text { 31. Problemas para } \\
\text { dormir }\end{array}$ & & .673 & \\
\hline $\begin{array}{l}\text { 4. Necesidad de analizar } \\
\text { la situación }\end{array}$ & & & .830 \\
\hline 6. Aprender de los errores & & & .802 \\
\hline $\begin{array}{l}\text { 2. Asumir la responsabili- } \\
\text { dad de mis actos }\end{array}$ & & & .785 \\
\hline Número de reactivos & 6 & 5 & 3 \\
\hline Varianza explicada & 36.94 & 14.22 & 7.76 \\
\hline Alpha de Cronbach & .82 & .83 & .74 \\
\hline Media & 3.247 & 3.154 & 4.697 \\
\hline Desviación Estándar & 1.23 & 1.32 & 1.14 \\
\hline
\end{tabular}

Para definir cada uno de los factores se tomó en consideración los reactivos que los conforman: Factor 1. Conflicto (6 reactivos). Se caracteriza por un alto índice de discusiones y mala comunicación que son fuente de estrés, consecuencia de la culpa en la relación. Factor 2. Ansiedad (5 reactivos). Consiste en respuestas emocionales que engloban aspectos subjetivos o cognitivos de carácter displacentero, fuentes de estrés, ante la experiencia de la culpa. Factor 3. Afrontamiento (3 reactivos). Se refiere a las conductas que realiza un integrante de la relación, para generar cambios tanto emocionales como psicológicos, dirigidos a eliminar la culpa. 
Subescala revaloración: la Tabla 6 muestra la solución factorial para la subescala revaloración, esta quedó conformada por 13 reactivos agrupados en tres factores que explican el $60.628 \%$ de la varianza total con un Alpha de Cronbach global de 0.828 y Alpha por factor entre 0.504 y 0.852 .

Tabla 6. Reactivos y factores de la subescala revaloración

\begin{tabular}{|c|c|c|c|}
\hline \multicolumn{4}{|c|}{ "Al pensar en la culpa que siento hacia mi pareja..." } \\
\hline \multirow{2}{*}{ Reactivos } & \multicolumn{3}{|c|}{ Factores } \\
\hline & 1 & 2 & 3 \\
\hline $\begin{array}{l}\text { 12. Pienso en los remordimientos } \\
\text { por haber lastimado a mi pareja }\end{array}$ & .853 & & \\
\hline $\begin{array}{l}\text { 8. Pienso en la desilusión por } \\
\text { el daño que le cause a mi pareja }\end{array}$ & .796 & & \\
\hline $\begin{array}{l}\text { 10. Pienso en el daño causado } \\
\text { a mi pareja }\end{array}$ & .761 & & \\
\hline $\begin{array}{l}\text { 11. Pienso en como compensar } \\
\text { a mi pareja el daño causado }\end{array}$ & .708 & & \\
\hline $\begin{array}{l}\text { 18. Pienso en la tristeza que } \\
\text { me causa }\end{array}$ & .673 & & \\
\hline 6. Pienso arrepentirme & .572 & & \\
\hline $\begin{array}{l}\text { 3. Reflexiono sobre lo que } \\
\text { hice mal }\end{array}$ & & .859 & \\
\hline 2. Aprendo de la experiencia & & .858 & \\
\hline $\begin{array}{l}\text { 1. Imagino cómo resolver la } \\
\text { situación }\end{array}$ & & .775 & \\
\hline 15. Analizo la situación & & .648 & \\
\hline $\begin{array}{l}\text { 4. Si se que actué mal, evito } \\
\text { pensar en ello }\end{array}$ & & & .750 \\
\hline $\begin{array}{l}\text { 14. Pienso que alejarme ayudaría } \\
\text { a mejorar la situación }\end{array}$ & & & .747 \\
\hline 9. Pienso en como sentirme mejor & & & .554 \\
\hline Número de reactivos & 6 & 4 & 3 \\
\hline Varianza explicada & 36.68 & 13.31 & 10.64 \\
\hline Alpha de Cronbach & .85 & .83 & .50 \\
\hline Media & 4.97 & 4.20 & 3.63 \\
\hline Desviación Estándar & .97 & 1.17 & 1.15 \\
\hline
\end{tabular}

La definición de los factores se presenta a continuación: Factor 1. Cogniciones negativas (6 reactivos). Se caracteriza por los pensamientos producto de las emociones desagradables o negativas generadas por sentir culpa. Factor 2. Autocorrección (5 reactivos). Se refiere a los pensamientos que las personas tienen después de haber causado daño a su pareja y que van encaminadas a mejorar o aprender de la situación.
Factor 3. Evitación (3 reactivos). Consiste en los pensamientos que tienen las personas sobre alejarse de la situación para evitar el malestar ocasionado por la culpa.

Subescala efectos de la culpa: la solución factorial para esta subescala, quedó conformada por 23 reactivos que se agruparon en tres factores que explican el $52.663 \%$ de la varianza total con un Alpha de Cronbach global de 0.807 y Alpha por factor que oscilan de 0.768 a 0.897 (ver Tabla 7).

Tabla 7. Reactivos y factores de la subescala efectos de la culpa

"Lo que hago cuando experimento culpa en mi relación de pareja es..."

\begin{tabular}{|c|c|c|c|}
\hline \multirow{2}{*}{ Reactivos } & \multicolumn{3}{|c|}{ Factores } \\
\hline & 1 & 2 & 3 \\
\hline 16. Aclaro los malos entendidos & .739 & & \\
\hline $\begin{array}{l}\text { 21. Me pongo en el lugar de mi } \\
\text { pareja }\end{array}$ & .699 & & \\
\hline $\begin{array}{l}\text { 29. Busco la reconciliación con mi } \\
\text { pareja }\end{array}$ & .694 & & \\
\hline 6. Busco la manera de remediarlo & .694 & & \\
\hline $\begin{array}{l}\text { 1. Hablo con mi pareja para resolver } \\
\text { la situación desagradable }\end{array}$ & .693 & & \\
\hline 7. Platico con mi pareja & .683 & & \\
\hline 13. Aprendo de la situación & .671 & & \\
\hline $\begin{array}{l}\text { 22. Recupero la confianza de mi } \\
\text { pareja }\end{array}$ & .654 & & \\
\hline 25. Escucho a mi pareja & .645 & & \\
\hline 9. Pido perdón a mi pareja & .629 & & \\
\hline 18. Pongo más atención a mi pareja & .626 & & \\
\hline 8. Me hago la victima & & .760 & \\
\hline 14. Chantajeo a mi pareja & & .706 & \\
\hline 35. Hacer sentir culpable a mi pareja & & .706 & \\
\hline 17. Huyo de la situación & & .686 & \\
\hline 20. Me alejo & & .665 & \\
\hline 5. Prefiero ignorar lo que sucede & & .638 & \\
\hline 2. Me enojo & & .608 & \\
\hline 10. Doy regalos a mi pareja & & & .821 \\
\hline 23. Le compro cosas & & & .804 \\
\hline 32. Soy detallista con mi pareja & & & .773 \\
\hline $\begin{array}{l}\text { 34. Compenso a mi pareja por el } \\
\text { daño causado }\end{array}$ & & & .640 \\
\hline 36. Soy complaciente con mi pareja & & & .625 \\
\hline Número de reactivos & 11 & 7 & 5 \\
\hline Varianza explicada & 29.05 & 16.17 & 7.45 \\
\hline Alpha de Cronbach & .89 & .768 & .838 \\
\hline Media & 4.66 & 2.52 & 3.44 \\
\hline Desviación Estándar. & .94 & 1.06 & 1.26 \\
\hline
\end{tabular}


Los factores se definieron de la siguiente manera: Factor 1. Reparación del daño (11 reactivos). Se refiere a todas las conductas consideradas necesarias para resarcir el daño causado a su pareja, con la finalidad de hacerla sentir mejor. Factor 2. Chantaje/manipulación (7reactivos). Consiste en las tácticas utilizadas por el trasgresor para atribuir la responsabilidad de los problemas y hacerle sentir culpable. Factor 3. Compensación (5 reactivos). Comprende las conductas dirigidas a pagar o compensar a la pareja por hacerle pasar un mal rato, con la finalidad de sentirse mejor. Las correlaciones entre los factores de cada una de las subescalas se obtuvieron con la correlación producto momento de Pearson, la mayoría de ellas fueron positivas y significativas (ver Tabla 8).

Tabla 8. Correlación de los factores de cada subescala de culpa

\begin{tabular}{|c|c|c|c|}
\hline $\begin{array}{l}\text { Significado de } \\
\text { culpa }\end{array}$ & $\begin{array}{l}\text { Nocivo/ } \\
\text { negativo }\end{array}$ & $\begin{array}{cc}\text { Valores } & \text { Autocrí- } \\
\text { tica }\end{array}$ & $\begin{array}{l}\text { Preocupación } \\
\text { empática }\end{array}$ \\
\hline Nocivo/negativo & 1 & & \\
\hline Valores & $.539^{* *}$ & 1 & \\
\hline Autocrítica & $.521^{* *}$ & $.457^{* *}$ & \\
\hline $\begin{array}{l}\text { Preocupación } \\
\text { empática }\end{array}$ & .084 & $.123^{\star *}$ & 1 \\
\hline $\begin{array}{l}\text { Causas de la } \\
\text { culpa }\end{array}$ & Falta de Com & npromiso & lencia \\
\hline $\begin{array}{l}\text { Falta de } \\
\text { compromiso }\end{array}$ & 1 & & \\
\hline Violencia & $.640^{*}$ & & 1 \\
\hline $\begin{array}{l}\text { Consecuen- } \\
\text { cias de la } \\
\text { culpa }\end{array}$ & Conflicto & Ansiedad & $\begin{array}{l}\text { Afronta- } \\
\text { miento }\end{array}$ \\
\hline Conflicto & 1 & & \\
\hline Ansiedad & $.661^{* *}$ & 1 & \\
\hline Afrontamiento & $.100^{* *}$ & $.143^{* *}$ & 1 \\
\hline Revaloración & $\begin{array}{l}\text { Emociones } \\
\text { negativas }\end{array}$ & Autocorrección & Evitación \\
\hline $\begin{array}{l}\text { Cogniciones } \\
\text { negativas }\end{array}$ & 1 & & \\
\hline Autocorrección & $.464^{\star *}$ & 1 & \\
\hline Evitación & $.148^{* *}$ & $.267^{* *}$ & 1 \\
\hline $\begin{array}{l}\text { Efectos de la } \\
\text { culpa }\end{array}$ & $\begin{array}{l}\text { Reparación } \\
\text { del daño }\end{array}$ & $\begin{array}{l}\text { Chantaje/ } \\
\text { Manipulación }\end{array}$ & $\begin{array}{l}\text { Compensa- } \\
\text { ción }\end{array}$ \\
\hline $\begin{array}{l}\text { Reparación del } \\
\text { daño }\end{array}$ & 1 & & \\
\hline $\begin{array}{l}\text { Chantaje/ } \\
\text { Manipulación }\end{array}$ & $-.338^{* *}$ & 1 & \\
\hline Compensación & $.393^{\star *}$ & $.119^{* *}$ & 1 \\
\hline
\end{tabular}

\section{Análisis factorial confirmatorio}

Para finalizar y con el objetivo de poner a prueba los resultados del análisis factorial del inventario multidimensional de culpa en la pareja, se realizó un AFC, mediante el programa AMOS 21 para SPSS. Para evaluar el ajuste del modelo se realizaron análisis de modelos estructurales, y se emplearon tres tipos de medidas de ajuste global, de ajuste absoluto y parsimonioso o incremental. Las medidas de ajuste absoluto evalúan el grado de exactitud en que el modelo predice correctamente la matriz de covarianza observada, cambio en Chi cuadrado de los modelos alternativos, el índice de ajuste comparativo de Bentler (CFI), el índice de ajuste normalizado de Bollen (NFI) y el error cuadrado de aproximación a las raíces medias (RMSEA). El estadístico Chi-cuadrado indica el ajuste absoluto del modelo, pero es muy sensible al tamaño de la muestra. Por lo que, usualmente se interpreta también la razón de Chi-cuadrado sobre los grados de libertad. Los índices CFI y NFI varían entre 0 y 1 , con 0 indican ausencia de ajuste y 1 ajuste óptimo; valores de 0.95 o superiores son considerados excelentes y valores por encima de 0.90 sugieren un ajuste aceptable del modelo a los datos. El índice RMSEA es considerado adecuado cuando sus valores son de 0.05 hasta 0.08 ( $\mathrm{Hu}$ \& Bentler, 1999, Jackson, 2007).

En este estudio se realizaron cinco análisis confirmatorios, uno para cada subescala del inventario multidimensional de culpa en la relación de pareja. En cada caso, se estimó el análisis factorial confirmatorio para corroborar la pertinencia de cada reactivo a las dimensiones halladas en el análisis factorial exploratorio.

\section{Resultados}

Se realizó un primer análisis para la subescala significado de culpa, los resultados mostraron que el modelo de los cuatro factores relacionados, no presentó buen ajuste. Puntualmente los indicadores de ajuste empleados mostraron los siguientes valores: $\mathrm{CMIND/DF}=4.047$, $\mathrm{CFI}=.889, \mathrm{NFI}=.849, \mathrm{IFI}=.888 \mathrm{y}$ RMSEA $=0.60$. Al seguir las indicaciones señaladas en la literatura para mejorar el ajuste del modelo, se examinó la matriz de residuos estandarizados (Kline, 2011). En un primer paso en la búsqueda de posibles soluciones 
que se traduzcan en un ajuste aceptable del modelo propuesto, se descartaron los reactivos $7,9,24$ y 1 . Los resultados mostraron los nuevos valores de los indicadores de ajuste: $\mathrm{CMIND} / \mathrm{DF}=3.925 ; \mathrm{CFI}=0.930$, $\mathrm{IFI}=0.931, \mathrm{NFI}=.908$ y $\mathrm{RMSEA}=0.057$, datos que indican un buen ajuste. Las cargas factoriales estandarizadas, betas y fiabilidad de cada factor se muestran en la Figura 1.

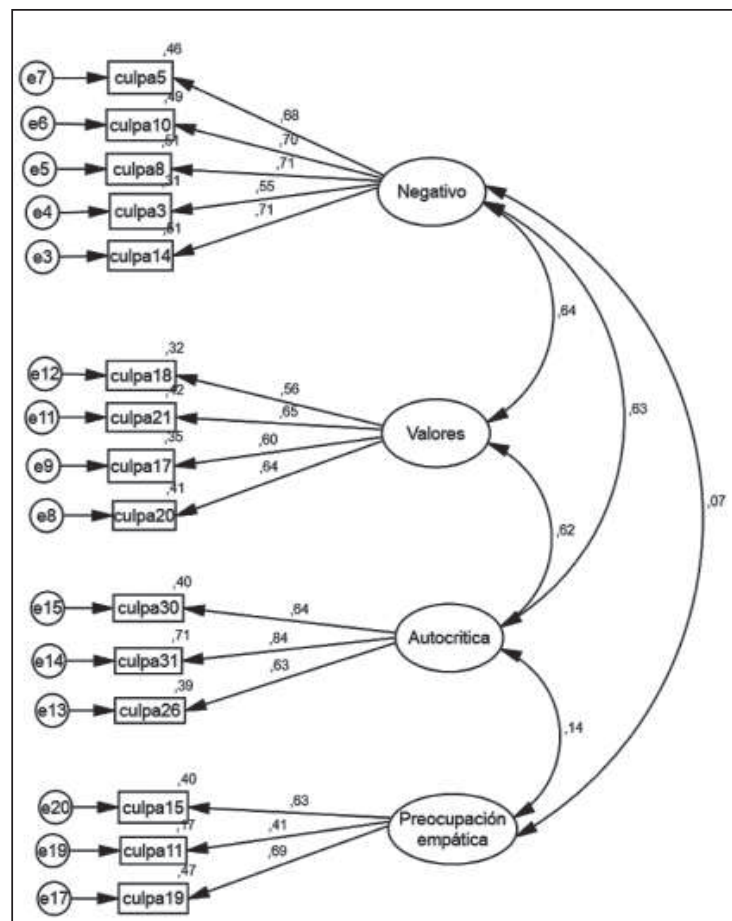

Figura 1. Modelo comprobatorio de la subescala significado de culpa en la relación de pareja

En segundo lugar se evaluó el ajuste del modelo de la subescala causas de la culpa que consta de dos factores, falta de compromiso integrado por 13 reactivos $(17,20,33,18$, $30,16,25,21,31,10,27,14,15)$ y el factor violencia, integrado por 3 reactivos $(1,2,3)$. El modelo de los dos factores relacionados correspondientes a esta subescala no presentó buen ajuste. Puntualmente los indicadores de ajuste empleados presentaron los siguientes valores: $\mathrm{CMIND} / \mathrm{DF}=6.969, \mathrm{CFI}=0.931$, $\mathrm{NFI}=8.97, \mathrm{IFI}=0.931$ y $\mathrm{RMSEA}=0.084$.
Al seguir las indicaciones señaladas en la literatura para mejorar el ajuste del modelo, se examinó la matriz de residuos estandarizados. A partir de los datos obtenidos se eliminaron los reactivos 16 y 31 , los resultados mostraron los nuevos valores de los indicadores de ajuste: $\mathrm{CMIND} / \mathrm{DF}=5.594 ; \mathrm{NFI}=0.944 ; \mathrm{CFI}=0.953$; $\mathrm{IFI}=0.953$ y $\mathrm{RMSEA}=0.074$, datos que indican un adecuado ajuste (ver Figura 2).

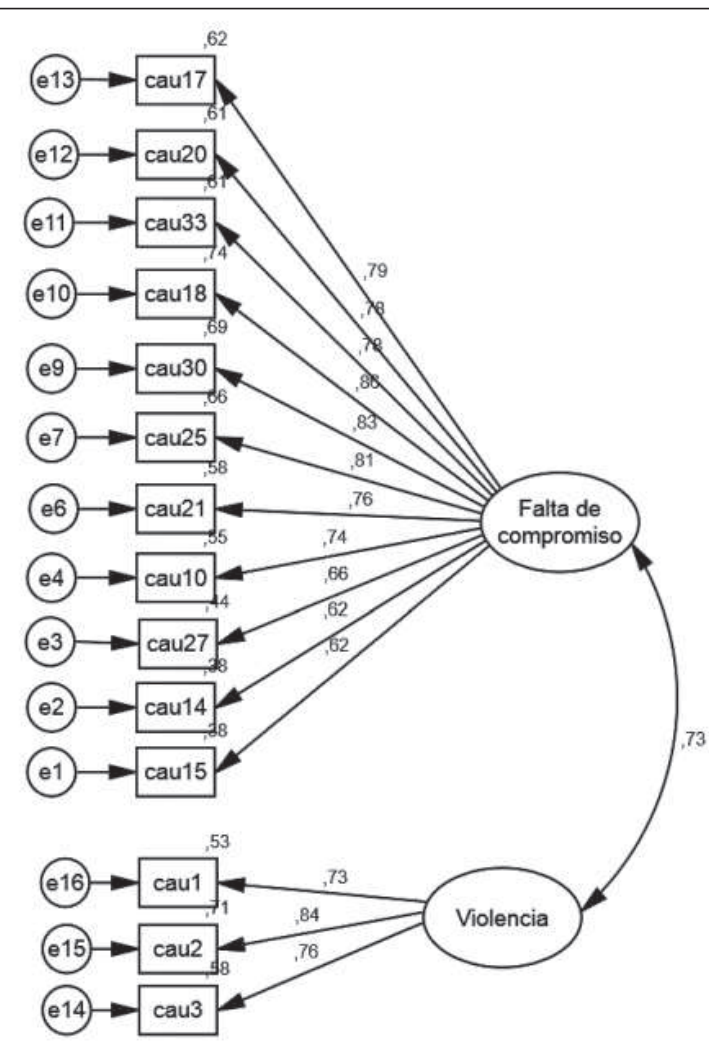

Figura 2. Modelo comprobatorio de la subescala causas de la culpa en la relación de pareja

La subescala consecuencias de la culpa: que consta de tres factores; conflicto, integrado por 6 reactivos $(11,9,7,13,20,26)$; el factor ansiedad integrado por 5 reactivos $(38,43,36,39$, $31)$, y el factor enfrentamiento con 3 reactivos (4, $6,2)$. Los resultados mostraron que el modelo de los tres factores relacionados presentó buen ajuste. Puntualmente los indicadores de ajuste empleados presentaron los siguientes valores: 
CMIND/DF = 3.417; NFI = 0.941; CFI = 0.957; $\mathrm{IFI}=0.957$ y RMSEA $=0.053$. Por lo que no fue necesario eliminar ningún reactivo (ver Figura 3).

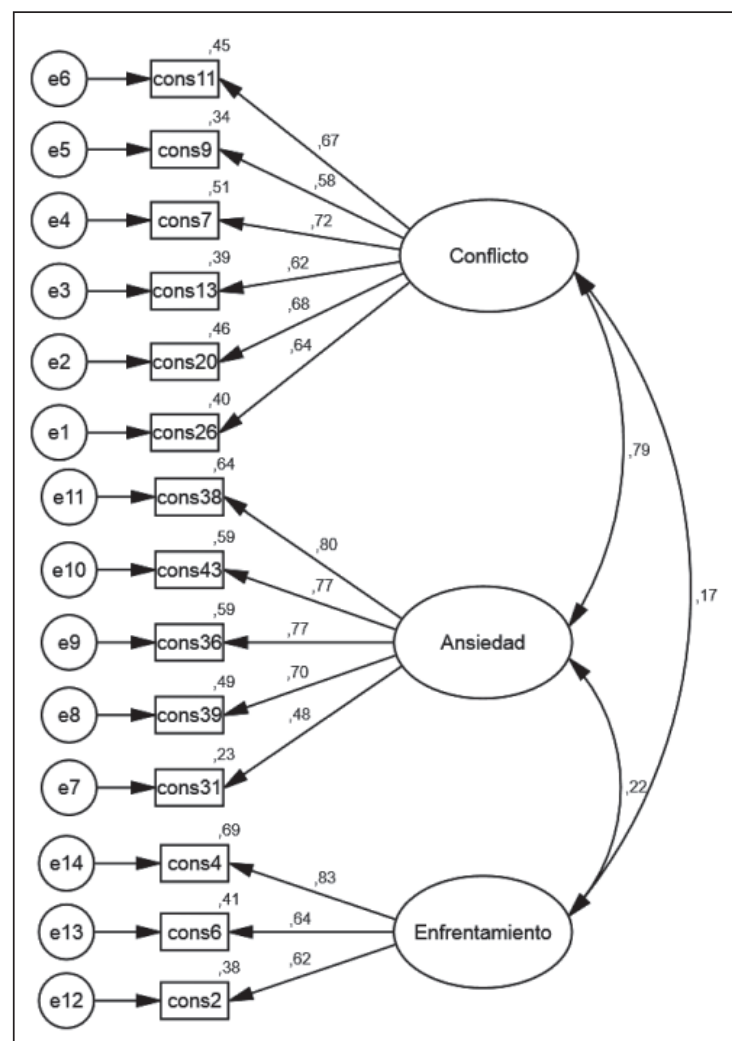

Figura 3. Modelo comprobatorio de la subescala consecuencias de la culpa en la relación de pareja

Se realizó un cuarto análisis factorial confirmatorio para evaluar el ajuste del modelo de la subescala revaloración, que consta de tres factores: cogniciones negativas integrado por 6 reactivos $(12,8,10,11,18,6)$; autocorrección integrado por 4 reactivos $(3,2,1,15)$ y evitación por 3 reactivos $(4,14,9)$.

Los resultados mostraron que el modelo, no presentó buen ajuste, puntualmente los indicadores de ajuste empleados presentaron los siguientes valores: CMIND/DF $=7.281$; $\mathrm{NFI}=0.889 ; \mathrm{CFI}=0.905, \mathrm{IFI}=0.905 \mathrm{y}$ RMSEA $=0.86$. Posteriormente se examinó de modo similar como en los análisis precedentes, para obtener mejora en el modelo propuesto. Se procedió a eliminar los reactivos 8, 18 y 15, los resultados mostraron los nuevos valores de los indicadores de ajuste: $\mathrm{CMIND/DF}=$ 6.286; $\mathrm{NFI}=0.927 ; \mathrm{CFI}=0.938 ; \mathrm{IFI}=0.938$, RMSEA $=0.077$ (ver Figura 4).

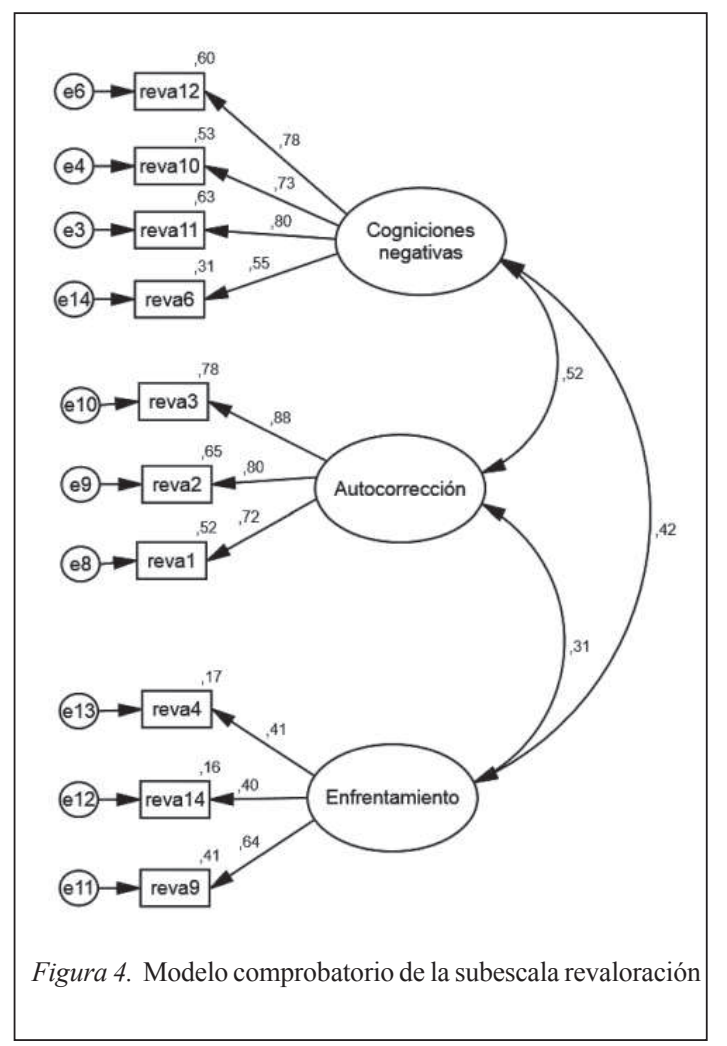

Se evaluó el ajuste del modelo de la subescala efectos de la culpa, que consta de tres factores; reparación del daño integrado por 11 reactivos $(16,21,29,6,1,7,13,22,25,9,18)$; chantaje/manipulación con 7 reactivos $(8,14$, $35,17,20,5,2)$ y compensación con 5 reactivos $(10,23,32,34,36)$. Para poder identificar el modelo se fijó en 1 el primer reactivo de cada factor.

El modelo no presentó buen ajuste. Puntualmente los indicadores de ajuste empleados presentaron los siguientes valores: $\mathrm{CMIND} / \mathrm{DF}=4.888 ; \mathrm{NFI}=0.873 ; \mathrm{CFI}=0.884$ $\mathrm{IFI}=0.885$ y $\mathrm{RMSEA}=0.068 \mathrm{Al}$ igual que en los análisis anteriores se examinaron los estimados, a fin de obtener posibles re-especificaciones que se traduzcan en un mejor ajuste del modelo. Se procedió a eliminar los reactivos, 2, $5,20,34,36$, los nuevos resultados mostraron los nuevos valores de los indicadores de ajuste: 
$\mathrm{CMIND} / \mathrm{DF}=4.312 ; \mathrm{NFI}=0.906 ; \mathrm{CFI}=0.926$; $\mathrm{IFI}=0.926$ y $\mathrm{RMSEA}=0.062$, los datos indican un adecuado ajuste del modelo (ver Figura 5).

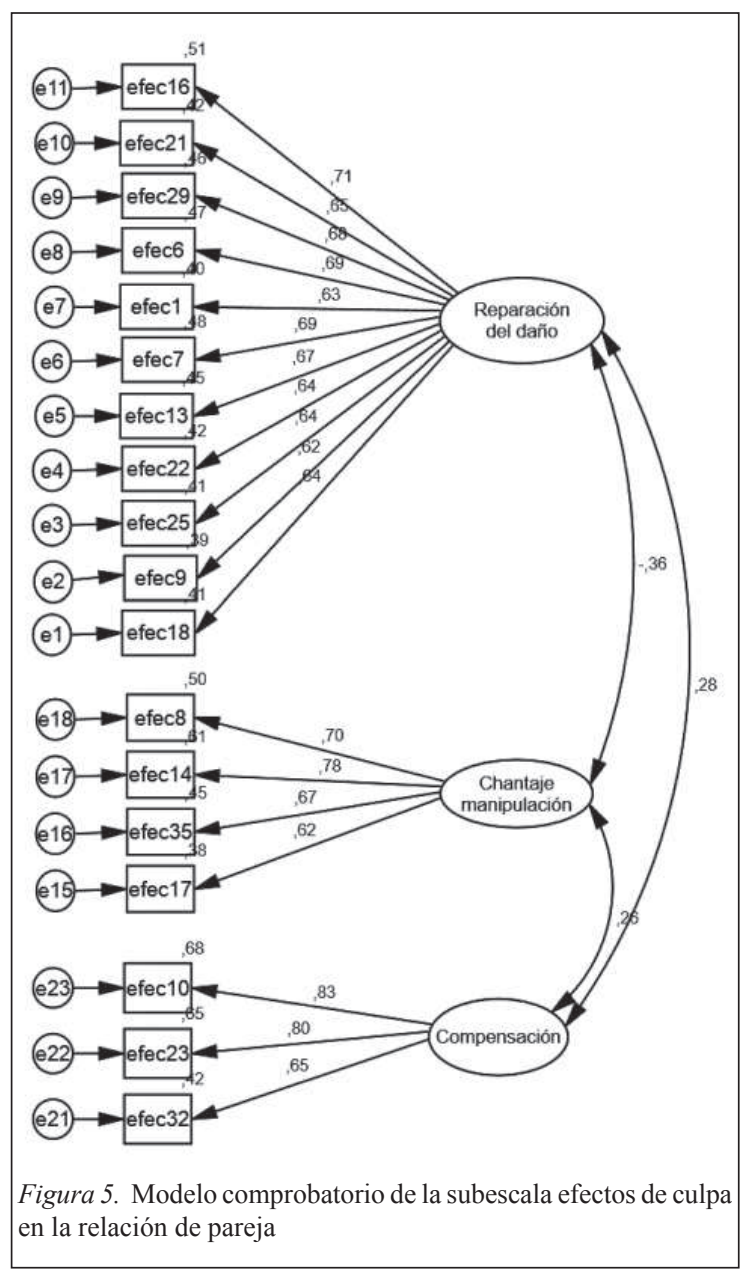

\section{Discusión}

En este estudio, se pudo comprobar que la culpa es una emoción que representa un papel determinante en las relaciones de pareja. Debido a que los sentimientos empáticos tienden a transformarse en culpa, cuando un integrante de la díada se considera responsable del sufrimiento del otro, motiva conductas dirigidas a la reparación del daño y a la autocorrección, lo que lleva a la mejora de sus relaciones. Estos aspectos se ven reflejados en la escala multidimensional de culpa que con sus subescalas y factores, discriminan adecuadamente entre los dos aspectos positivos y negativos (Álvarez, 1996).

En la subescala significado de la culpa, se concluyó que ésta es percibida en la relación de pareja como algo negativo, que va en contra de sus valores y que genera malestar al reflexionar sobre el daño causado a la pareja. También se hizo evidente el componente empático, pues las personas reportaron que es importante preocuparse por el bienestar de la pareja y consideran necesario buscar la manera de reparar el daño causado a su compañero/a. Cómo ya lo refería Frijda (1994), la culpa es en su origen negativa, pero en su función resulta positiva, de acuerdo con Baumeister et al. (1994) esto se da a través de conductas reparadoras del daño, que la transforman en una emoción conciliadora en la interacción de pareja.

Si bien el proceso empático es de suma importancia, para que éste ocurra es necesario que haya un entendimiento emocional, pues permite a las personas comprender la complejidad de sus emociones. Además, conocer las causas y consecuencias dan como resultado expresiones emocionales cultural y socialmente acorde con las normas establecidas y de este modo favorecen las interacciones personales (Sánchez-Aragón, Retana \& Carrasco, 2008).

En la subescala causas de la culpa, se puede observar que las más frecuentes tienen que ver con situaciones en las que perciben el sufrimiento de su compañero/a como consecuencia de sus acciones, durante la interacción diaria. Principalmente se refiere a contextos relacionados con la violencia y la falta de compromiso por parte de uno de los miembros de la pareja. Estas acciones ocasionan daño, pero además generan malestar en el agresor, por considerarse el causante del dolor de su compañero/a.

Se puede concluir que se encontró evidencia suficiente en los resultados para confirmar lo propuesto por la teoría respecto a que las principales causantes de culpa tienen que ver con el descuido de las relaciones y/o el incumplimiento del compromiso con la pareja (Baumeister et al., 1995; Hoffman, 2002). Los hallazgos sugieren que la falta de compromiso y la violencia engloban situaciones como, evitar escuchar a la pareja, dañarla, dedicarle poco tiempo, entre otras. Asimismo, la violencia puede ser pasiva y manifestarse como agresión contenida, por ejemplo al ignorar a la pareja, evitarla, rechazarla; acciones que permiten observar el componente agresivo de la culpa.

Respecto a la subescala consecuencias de la culpa, los datos obtenidos indican que la culpa ocasiona principalmente conflicto y ansiedad en la pareja. Sugiere que al desconocer la forma 
de resolver la situación generadora de estrés y malestar, los integrantes de la relación amorosa tienden a reaccionar con enojo y terminan en discusiones, situación que es atribuida también a una comunicación inadecuada. De esta manera, el resultado de experimentar esta emoción lleva a las personas a buscar alternativas para solucionar la situación y de alguna manera eliminar la sensación de ansiedad (Álvarez, 1996; Etxebarria, 2006). Se puede argumentar que, esto se da a partir de las conductas de Afrontamiento tales como la reflexión, analizar que se hizo mal y la necesidad de resolver la situación, lo que lleva a las personas a asumir la responsabilidad de sus acciones y aprender de sus errores. Esto es apoyado en lo que explican algunos autores respecto a que la culpa mal dirigida genera conflicto en la interacción de pareja (Horan \& Dillow, 2009; Valor Segura, Expósito \& Moya, 2010) situaciones que hacen evidente el componente ansioso-agresivo de la culpa (Etxebarria, Ortíz, Conejero \& Pascual, 2009).

Con relación a lo que piensan cuando sienten culpa, la subescala de revaloración, apunta a que los miembros de la pareja reflexionan sobre las emociones negativas que les provoca, pensar en que hicieron mal, en acciones de corrección y en cómo evitar esta experiencia, como resultado de sentir remordimiento, tristeza y arrepentimiento por el daño causado a su pareja (Álvarez, 1996; Etxebarria \& Apodaca, 2008; Frijda, 1993).

Se sugiere que las conductas de autocorrección surgen al estar expuestos a estas emociones negativas, que causan molestar y daño emocional, y el intento de liberarse de éstas los lleva a la modificación o evitación de conductas que causan daño a la pareja. Es importante señalar que la evitación no solo es una estrategia para reducir los problemas, sino que también se puede atribuir a la incapacidad o falta de asertividad para resolverlos, que además puede ser utilizada para ignorar lo que sucede, mantenerse al margen de la situación y no adquirir la responsabilidad que le corresponde respecto al estado de la relación. Por tanto, al no alejarse y recurrir a la autocorrección moral cumplen con el rol sociocultural establecido, ya que viven bajo la presión social del medio donde se desarrolla como ya lo había mencionado Etxebarria (2000).

Finalmente, la subescala de efectos de la culpa permitió conocer qué es lo que las personas mencionan como acciones para enmendar el daño que causan a su pareja. Se identificaron tres factores, uno positivo que se refiere a la re- paración del daño, el cual tiene como finalidad resolver situaciones conflictivas y remediar el sufrimiento de su compañero/a. De acuerdo con los participantes, esto se realiza a través de conductas como, platicar para resolver la situación, ponerse en el lugar del otro, demostrarle afecto, pedir perdón, recuperar la confianza y ser más atento con la pareja. Estas conductas van dirigidas específicamente a procurar que el otro se sienta mejor y recupere su estabilidad emocional, pues como ya lo proponían Etxebarria (1999) y Tangney (1991) las acciones reparadoras promueven la buena comunicación y la mejora de las relaciones.

Sin embargo, los otros dos factores comúnmente buscan el beneficio propio, por ejemplo, la compensación que trata de reparar la mala conducta a través de regalos o situaciones frívolas, además de mostrarse complaciente con la finalidad de que la pareja se sienta mejor, aunque en el fondo no le interese su bienestar emocional. Otro aspecto negativo de esta subescala es la manipulación y el chantaje, estas conductas son utilizadas por el/la culpable como táctica de poder, debido a que es probable que presente comportamientos relacionados con querer influenciar en la pareja para tratar de atribuirle la responsabilidad de los conflictos, además podría utilizar estas estrategias como forma de castigo (Rivera Aragón \& Díaz Loving, 2002). De esta manera, se favorece la eliminación de la culpa y se podría mantener una relación de poder en donde la compensación, además del chantaje y la manipulación, resuelven a medias los conflictos para mantener la relación estable aunque no haya mejora como ya lo mencionaban Etxebarria et al. (2010).

\section{Conclusiones}

Los resultados mostraron evidencia para concluir, que a pesar de tratarse de un constructo difícil de abordar por su carácter negativo y dañino, aunado al factor de la deseabilidad social, los análisis estadísticos realizados permiten concluir que se obtuvo una escala válida y confiable para medir la culpa en cinco de sus dimensiones: significado, causas, consecuencias, cogniciones y efectos. Esta escala discrimina entre los aspectos negativos que causan malestar y los positivos representados primordialmente por su carácter empático y reparador. Además se puede resaltar que por lo menos en esta muestra, la culpa es percibida como parte de la interacción 
de la pareja y que tiene efectos tanto negativos como positivos. Estos efectos motivan a los individuos a la autorreflexión y autocorrección de conductas, con el fin de resolver y evitar los conflictos dentro de la vida en pareja, para mejorar la relación y quizá a través de la reparación y compensación, establecer relaciones más satisfactorias.

\section{Referencias}

Álvarez, G. N. (1996). La tendencia a sufrir el castigo: una contribución a la teoría de la culpa. Alcalá Henares: Universidad de Álcala de Henares.

Barrett, K. C. (1995). A Funcionalist approach to shame and guilt. In J. P. Tangney \& K. W. Fischer (Eds.), Selfconscious emotions: Shame, guilt, embarrassment, and pride (pp. 25-63). Nueva York: Guilford.

Baumeister, R., Reis, H., \& Delespaul, P. (1995). Subjective and experimental correlates of guilt in daily life. Personality and social Psychology Bulletin, 21(12), 1256-1268.

Baumeister, R., Stillwell, A., \& Heatherton, T. (1994) Guilt: An interpersonal approach, Psychological Bulletin, 115(2), 243-267.

Benetti-McQuoid, J., \& Bursik, K. (2005). Individual differences in experiences of and Responses to Guilt and Shame: Examining the Lenses of Gender and Gender Role. Sex Roles, 53(1-2), 133-142.

Berrios, G. E., Bulbena, A., Bakshi, N., Dening, T. R., Jenaway, A., Markar, H., ... Mitchell, S. L. (1992). Feelings of guilt in major depression: Conceptual and psychometric aspects. British Journal of Psychiatry, 160, 781-787.

Buss, A. H. \& Durkee, A. (1957). An inventory for assessing different kinds of hostility. Journal of Consulting Psychology. 21(4) 343-349.

Caprara, G. V., Manzi, J. \& Perugini, M. (1992). Investigating guilt in relation to emotionality and aggression. Personality and Individual Differences, 13(5), 519-532.

Etxebarria, I. (1999). Psicología moral y crecimiento personal. Barcelona: Ariel Psicología

Etxebarria, I. (2000). Guilt: an emotion under suspicion. Psicothema, 12(11), 101-108.

Etxebarria, I (2006). Los sentimientos de culpa: ¿Qué hacemos con ellos? Málaga: Arguval.

Etxebarria, I. \&Apodaca, P. (2008). Both Freud and Hoffman are right: Anxious-aggressive and empathic dimensions of guilt. The Spanish Journal of Psychology, 11(1), 159-171.

Etxebarria, I., Ortiz, M. J., Conejero, S. \& Pascual, A. (2009). Intensity of habitual guilt in men and women: Differences in interpersonal sensitivity and the tendency towards anxious-aggressive guilt. The Spanish Journal of Psychology, 12(2), 540-554.

Etxebarria, I., Pascual, A., \& Conejero, S. (2010) ¿Puede considerarse la culpa un índice de sensibilidad interpersonal? Reactividad interpersonal y culpa interpersonal. Ansiedad y estrés, 16(2-3), 201-214.

Etxebarria, I., \& Pérez, J. (2003). ¿Qué nos hace sentir culpa? Categorías de eventos en adolescentes y adultos de uno y otro sexo. Estudios de Psicología, 24(2), 241-252.

Evans, D. R., Jessup, B. A., \& Hearn, M. T. (1975). Development of a reaction inventory to measure guilt. Journal of Personality Assessment, 39, 421-423.
Fernández de Ortega, B. H. \& Reidl, M. L. (2005) Adaptación del inventario de culpa rasgo-estado y estándares morales para la muestra mexicana de Jones, Schratter \& Kugler. Revista Mexicana de Psicología, 22 (2), 513-517

Frijda, N. H. (1993). The place of appraisal in emotion. En N. H. Frijda (Ed.), Appraisal and Beyond. The issue of cognitive determinants of emotion (pp. 357-387). Hove, UK: Erlbaum.

Frijda, N.H. (1994). Emotions are functional, most of the time. En P. Ekman \& R.J. Davidson (Eds.), The nature of emotion: Fundamental questions (pp. 112-122). New York: Oxford University Press.

Hoffman, M. L. (1998). Varieties of empathy-based guilt. En J. Bybee (Ed.), Guilt and children (pp. 91 112). San Diego, CA: Academic Press.

Hoffman, M. L. (2002). Desarrollo moral y empatía: implicaciones para la atención y la justicia. Barcelona: Idea Books.

Horan, S.M. \& Dillow, M.R. (2009). Deceivers and emotion: The relationships among relational commitment, satisfaction, deceptive message type, and feelings of guilt and shame. The Atlantic Journal of Communication, 17(4), 149-165.

Hu, L., \& Bentler, P. M. (1999). Cutoff Criteria for Fit Indexes in Covariance Structure Analysis: Conventional Criteria Versus New Alternatives. Structural Equation Modeling, $6(1), 1-55$.

Jackson, D. L. (2007). The effect of the number of observations per parameter in misspecified confirmatory factor analytic models. Structural Equation Modeling: A Multidisciplinary Journal, 14(1), 48-76.

Klass, E. T. (1987). Situational approach to assessment of guilt: Development and validation of a self-report measure. Journal of Psychopathology and Behavioral Assessment, 9(1), 35-48.

Kline, R. (2011). Principles and Practice of Structural Equiation Modeling (3a. ed.). New York: The Guilford Press.

Kubany, E. S., Haynes, S. N., Abueg, F. R., Manke, F. P., Brennan, J. M., \& Stahura, C. (1996). Development and validation of the Trauma-Related Guilt Inventory (TRGI). Psychological Assessment, 8(4), 428-444.

Kugler, K., \& Jones, W. H. (1992). On conceptualizing and assessing guilt. Journal of Personality and Social Psychology, 62(2), 318-327.

Martorelli, C., González, R., Ordoñez, A., \& Gómez, O. (2011). Estudio comparativo del cuestionario de conducta prosocial (CCP) y su relación con variables de personalidad y socialización. RIDEP 32(2), 35-52.

Mosher, D. L. (1966). The development and multitraitmultimethod matrix analysis of three measures of three aspects of guilt. Journal of Consulting Psychology, $30(1), 25-29$.

O'Connor, L. E., Berry, J. W., Weiss, J., Bush, M. \& Sampson, H. (1997). Interpersonal guilt: The development of a new measure. Journal of Clinical Psychology, 53(1), 73-89.

Ojeda, G. A., Torres, G. T. \& Moreira, M. M. (2010). ¿Amor y compromiso en la pareja?: de la teoría a la práctica. RIDEP 30 (2), 125-142.

Otterbacher, J. R. \& Munz, D. C. (1973). State-trait measure of experiential guilt. Journal of Consulting and Clinical Psychology, 40(1), 115-121.

Reidl, L. \& Jurado, S. (2007). Culpa y vergüenza. México: Universidad Nacional Autónoma de México.

Reyes Lagunes, I. (1993), Redes semánticas para la construcción de instrumentos. Revista de Psicología Social y Personalidad, 9 (1), 81-93. 
Rivera Aragón, S., \& Díaz Loving, R. (2002). La cultura del poder en la pareja. México: Miguel Ángel Porrúa.

Rojas Marcos, L. (2009). Elsentimiento de culpa. España:Aguilar.

Sánchez Aragón, R., Retana Franco, B. E., \& Carrasco, C. E. (2008). Evaluación Psicológica del Entendimiento Emocional: Diferencias y Similitudes entre Hombres y Mujeres. RIDEP 26(2), 193-216.

Tangney, J. P. (1990). Assessing individual differences in proneness to shame and guilt: Development of the Self-Conscious Affect and Attribution Inventory. Journal of Personality and Social Psychology, 59(1), 102-111.

Tangney, J. P. (1991). Moral affect: the good, the bad, and the ugly. Journal of Personality and Social Psychology, 61(4), 598-607.

Tangney, J. P., Burggraf, S. A., \& Wagner, P. E. (1995) Shame-proneness, guilt-proneness, and psychological symptoms. En J. P. Tangney \& K. W. Fischer (Eds.), Self-conscious emotions: shame, guilt, embarrassment, and pride (pp. 243-267). New York: Guilford Press.
Tangney, J. P. (1995a). Recent advances in the empirical study of shame and guilt. American Behavioral Scientist, 38(8), 1132-1145.

Tangney, J. P. (1995b). Shame and guilt in interpersonal relationships. En J. P. Tangney \& K. W. Fischer (Eds.), Selfconscious emotions: Shame, guilt, embarrassment, and pride (pp. 114-139). New York: Guilford Press.

Tangney, J. \& Fischer, K. (1995). Self-conscious emotions. The psychology of shame, guilt, embarrassment, and pride. Nueva York: The Guilford Press.

Tangney, J. P. \& Dearing, R. L. (2004). Shame and guilt. Nueva York: The Guilford Press.

Thompson, T., Dinnel, D. L. \& Dill, N. J. (2003). Development and validation of a Body Image Guilt and Shame Scale. Personality and Individual Differences, 34, 59-75.

Valor Segura, I., Expósito, F. \& Moya, M. (2010). Powerful and powerless emotions in partner conflicts: Gender differences. Intervención Psicosocial, 2(19), 129-134.

Zabalegui, R. L. (1997). ¿Por qué me culpabilizo tanto? Bilbao: Descleé de Brower.

Para citar este artículo:

Alvarez Ramírez, E., García Méndez, M., \& Rivera Aragón, S. (2014). Medición de la culpa en la relación de pareja. Ciencias Psicológicas VIII (2): 115 - 128 